\title{
Low expression of microRNA-202 is associated with the metastasis of esophageal squamous cell carcinoma
}

\author{
GUOLIANG MA ${ }^{1}$, FENGMEI ZHANG ${ }^{2}$, XUEGUANG DONG $^{1}$, XIAOLI WANG ${ }^{1}$ and YUGUO REN $^{1}$ \\ Departments of ${ }^{1}$ Clinical Laboratory and ${ }^{2}$ Endocrinology, Laiwu People's Hospital, Laiwu, Shandong 271199, P.R. China
}

Received November 8, 2014; Accepted November 25, 2015

DOI: $10.3892 /$ etm.2016.3014

\begin{abstract}
The present study aimed to determine the expression levels and biological functions of microRNA-202 (miR-202) in patients with esophageal squamous cell carcinoma (ESCC). A total of 60 patients with ESCC and 30 healthy individuals were enrolled and reverse transcription-quantitative polymerase chain reaction was performed to measure the expression levels of miR-202. In order to investigate the effects of miR-202 expression levels on the proliferative, migratory and invasive abilities of ESCC cells, methylthiazolyl-tetrazolium bromide proliferation, in vitro scratch and Transwell ${ }^{\circledR}$ chamber assays were performed. Expression levels of miR-202 were significantly decreased in the peripheral blood of patients with ESCC, which is associated with the degree of cell differentiation and lymph node metastasis $(\mathrm{P}<0.05)$. Following miR-202 transfection, cell proliferation was significantly inhibited $(\mathrm{P}<0.05)$. Cell migration and invasion was also significantly inhibited by miR-202 transfection $(\mathrm{P}<0.05)$. The results of the present study demonstrated that the expression of miR-202 inhibited the proliferation, migration and invasion of ESCC cells. Furthermore, low expression levels of miR-202 were detected in the peripheral blood of patients with ESCC, which is associated with the development, invasion and metastasis of ESCC.
\end{abstract}

\section{Introduction}

Esophageal carcinoma is a common type of malignant tumor that forms in the upper digestive tract. Based on worldwide incidence rates, esophageal carcinoma is the ranks eighth most common type of malignant tumor, and has the sixth highest mortality rate of all types of cancer (1). Esophageal carcinoma is insidious at onset and early diagnosis is difficult. Furthermore, due to its ability to rapidly metastasize to

Correspondence to: Dr Yuguo Ren, Department of Clinical Laboratory, Laiwu People's Hospital, 1 Xuehu Street, Laiwu, Shandong 271199, P.R. China

E-mail:ryg000@163.com

Key words: esophageal squamous cell carcinoma, microRNA-202, invasion, metastasis the lymph nodes, the prognosis for esophageal carcinoma is poor (2). Esophageal carcinomas are subdivided into esophageal squamous cell carcinoma (ESCC) and adenocarcinoma, according to the tissue type. ESCC is more common in the developing world. In China, $>90 \%$ of all esophageal carcinomas are ESCC. With the application and development of surgery, chemotherapy, and immunotherapy, the prognosis for patients with ESCC has markedly improved. However, five-year survival rates for patients with ESCC remain low at $\sim 10 \%$, due to the invasive and metastatic potential of ESCC $(3,4)$. Earlier diagnosis of ESCC may reduce the probability of tumor cell metastasis and markedly improve the prognosis of patients $(5,6)$. Therefore, the identification of novel molecular markers for ESCC has potential clinical application for the early diagnosis of patients with ESCC.

MicroRNA (miRNA), which are small endogenic non-coding RNA molecules (18-22 nt), regulate gene expression by binding to 3'-untranslated regions of target mRNA $(7,8)$. The application of high-throughput sequencing and microarray technology has produced notable alterations in the miRNA expression profiles of tumor tissue, as compared with normal tissue $(9,10)$. Variations in miRNA expression levels between tumor tissues and normal tissues may lead to the dysfunction of downstream signaling pathways, which are associated with proliferation, invasion, angiogenesis and the metastasis of tumors $(11,12,13)$. Furthermore, miRNA molecules are highly stable, which facilitates their survival in tissue fluid, blood and urine (14). Therefore, miRNA has been demonstrated to be a suitable biomarker for the diagnosis of tumors.

MicroRNA-202 (miR-202) is a recently identified tumor miRNA molecule with various biological functions (15). The expression levels of miR-202 are associated with the proliferation, invasion, metastasis, apoptosis and angiogenesis of numerous tumor tissue types, including gastric, liver and pancreatic cancer (16-19). Analysis of the expression levels of genes in ESCC tissue, using the database of Gene Expression Omnibus (GSE20347), indicated that 1,755 genes were differentially expressed in ESCC tissues, including miR-202 (20). However, the expression levels and biological function of miR-202 in peripheral blood remains unclear. In the present study, the expression levels of miR-202 in the peripheral blood of patients with ESCC were analyzed using reverse transcription-quantitative polymerase chain reaction (RT-qPCR). In addition, the effects of miR-202 on cellular proliferation, migration and invasiveness were evaluated. 


\section{Materials and methods}

Reagents. TRIzol ${ }^{\circledR}$ used for RNA extraction was purchased from Invitrogen (Thermo Fisher Scientific Inc., Waltham, MA, USA). Takara PrimeScript ${ }^{\mathrm{TM}}$ RT Reagent kit and SYBR $^{\circledR}$ PrimeScript ${ }^{\mathrm{TM}}$ RT-PCR Kit II (Perfect Real Time) were purchased from Takara Biotechnology Co., Ltd. (Tokyo, Japan). Transwell ${ }^{\circledR}$ chambers were purchased from Corning Life Sciences (Lowell, MA, USA).

Patients. In the present study, ESCC tissues and peripheral blood samples were harvested from 60 patients with ESCC (38 men and 22 women) recruited from the Laiwu People's Hospital (Laiwu, China). Patients had not received chemotherapy or anti-tumor therapy and were aged between 38.5 and 67 years (average age, 52.6 years). A total of 29 cases of well differentiated, 22 cases of moderately differentiated and 9 cases of poorly differentiated cells were included in the ESCC tissue samples. Lymph node metastases were categorized as follows: N0, no evidence of lymph node metastasis; and N1, metastasis to lymph node. In the present study, patients were separated into $\mathrm{N} 1$ lymph node metastases $(\mathrm{n}=36)$ and N0 lymph node metastasis $(n=24)$ groups. For a control group, 30 healthy individuals were enrolled. For patients with ESCC, peripheral blood $(5 \mathrm{ml})$ was collected from the cubital vein one week prior to the surgical procedure. For healthy controls, peripheral blood $(5 \mathrm{ml})$ was collected from the cubital vein when the patients underwent physical examination. Peripheral blood samples were stored at $-80^{\circ} \mathrm{C}$ for further analysis. ESCC tissue samples were collected from the 60 patients with ESCC during surgery and maintained at $-80^{\circ} \mathrm{C}$ for further analysis. Prior written and informed consent was obtained from every patient. The present study was approved by the Ethics Review Board of Laiwu People's Hospital.

Cell culture and transfection. EC9706 ESCC cell lines were cultured in RPMI-1640 medium supplemented with Gibco $10 \%$ fetal bovine serum (FBS; Thermo Fisher Scientific Inc.), $100 \mathrm{U} / \mathrm{ml}$ penicillin and $100 \mu \mathrm{g} / \mathrm{ml}$ streptomycin (both obtained from Beyotime Institute of Biotechnology, Shanghai, China) in a humidified atmosphere containing $5 \% \mathrm{CO}_{2}$ at $37^{\circ} \mathrm{C}$. Cells in the logarithmic growth phase were used in the present study. Transfection of miR-202 mimics (Guangzhou RiboBio Co., Ltd., Guangzhou, China) was performed in $70 \%$ confluent cells using Lipofectamine ${ }^{\circledR} 2000$ reagent (Invitrogen; Thermo Fisher Scientific Inc.), according to the manufacturer's protocol. MiR-202 independent sequence was used as the negative control.

$R T$-qPCR analysis. Total RNA was extracted from peripheral blood samples using TRIzol ${ }^{\circledR}$ reagent. RNA quality was assessed using RNA electrophoresis and a spectrophotometer at optical density (OD) 260/280. RNA was reverse transcribed into cDNA using the PrimeScript ${ }^{\mathrm{TM}}$ RT Reagent kits. RT-qPCR was conducted using SYBR ${ }^{\circledR}$ PrimeScript $^{\mathrm{TM}}$ RT-PCR kit II (Perfect Real Time), according to the manufacturer's protocol. U6 was used as an internal control for normalization. PCR reactions were repeated $\geq 3$ times for each sample. Oligonucleotide primers of miR-202 and U6 were synthesized by Guangzhou RiboBio Co., Ltd. (Guangzhou, China), as follows: miR-202, forward 5'-CCTCCCAGGCTCACGAGG CT-3' and reverse 5'-GGTGCAGGTGCACTGGTGCA-3'; and U6, forward 5'-CAAAGTCAGTGCAGGTAGGCTTA-3' and reverse 5'-AACGCTTCACGAATTTGCGT-3'. The RT-qPCR reaction system included $10 \mu \mathrm{l}$ qRT-PCR-Mix, $0.5 \mu \mathrm{l}$ forward primer, $0.5 \mu 1$ reverse primer, $2 \mu \mathrm{l}$ cDNA and $7 \mu 1 \mathrm{ddH}_{2} \mathrm{O}$. The RT-qPCR was performed using an ABI 7300 thermal cycler (Applied Biosystems Inc., Foster City, CA, USA). The reaction procedure was as follows: Pre-denaturation at $95^{\circ} \mathrm{C}$ for $10 \mathrm{~min}$ and 40 cycles of $95^{\circ} \mathrm{C}$ for $1 \mathrm{~min}$ and $60^{\circ} \mathrm{C}$ for $30 \mathrm{sec}$. The $2^{-\Delta \Delta C Q}$ method (21) was used to analyze the relative expression levels of miR-202.

Methylthiazolyl-tetrazolium bromide (MTT) proliferation assay. EC9706 cells in the logarithmic phase were routinely digested for $12 \mathrm{~min}$ at $37^{\circ} \mathrm{C}$ using $0.2 \%$ trypsin/EDTA (Beyotime Institute of Biotechnology) and prepared as single-cell suspensions containing $5 \times 10^{5}$ cells $/ \mathrm{ml}$. Each well of a 96-well plate was seeded with $200 \mu \mathrm{l}$ cell suspension $\left(2 \times 10^{3}\right.$ cells/well). All 96-well plates were incubated at $37^{\circ} \mathrm{C}$ in an atmosphere containing $5 \% \mathrm{CO}_{2}$ for $0,24,48$ or $72 \mathrm{~h}$, after which the culture medium was discarded and replaced with $180 \mu 1$ RPMI-1640 medium and $20 \mu 1$ MTT solution (5 g/l; Beyotime Institute of Biotechnology). Following incubation at $37^{\circ} \mathrm{C}$ in an atmosphere containing $5 \% \mathrm{CO}_{2}$ for a further $4 \mathrm{~h}$, the supernatant was discarded, each well was supplemented with $150 \mu 1 /$ well dimethyl sulphoxide (Sigma-Aldrich, St. Louis, MO, USA) and the plates were shaken for 10 min using a shaking table concentrator (Molecular Devices LLC, Sunnyvale, CA, USA). The OD of each well was determined using a FlexStation ${ }^{\circledR} 3$ Multi-Mode Microplate Reader (Molecular Devices LLC) at $490 \mathrm{~nm}$. Tests were repeated in triplicate for each group.

In vitro scratch assay. Each well of a 24-well plate was seeded with $800 \mu 1 \mathrm{EC} 9706$ cell suspension $\left(2 \times 10^{3}\right.$ cells/well) and incubated for $24 \mathrm{~h}$ at $37^{\circ} \mathrm{C}$ in an atmosphere containing $5 \% \mathrm{CO}_{2}$. Once a confluent monolayer was formed, cells were serum-starved for $24 \mathrm{~h}$ and the cell monolayers were subsequently scratched using a $1,000-\mu 1$ pipette tip. Scratched cells were cultured in RPMI-1640 medium supplemented with 10\% FBS for $24 \mathrm{~h}$ and observed under an inverted microscope (Olympus BX50; Olympus Corporation, Tokyo, Japan; magnification, x10). The migratory ability of the cells was assessed by comparing the respective repair distances.

Transwell ${ }^{\circledR}$ chamber assay. Metastasis and invasive ability of the EC9706 cells was examined using a Transwell ${ }^{\circledR}$ chamber assay. Matrigel (BD Biosciences, Franklin Lakes, NJ, USA) was hydrated with serum-free medium (dilution, 1:2) and added to the upper chambers for $\geq 1 \mathrm{~h}$ at $37^{\circ} \mathrm{C}$ in a $\mathrm{CO}_{2}$ incubator. Following rehydration of the Matrigel, the lower chamber was filled with $500 \mu 1$ RPMI-1640 medium supplemented with $10 \%$ FBS and the upper chamber was filled with cell suspension diluted to $1 \times 10^{5}$ cells and $200 \mu 1$ serum-free RPMI-1640 medium. The invasion chamber plate was incubated for $24 \mathrm{~h}$. Cells invading through the Matrigel were fixed with $4 \%$ formaldehyde and subjected to Giemsa staining (Beyotime Institute of Biotechnology). These processes were all performed at $4^{\circ} \mathrm{C}$. Five fields were randomly selected from each section and the number of cells that invaded the Matrigel 
A

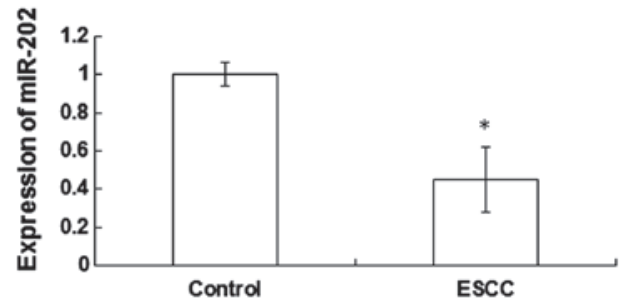

B

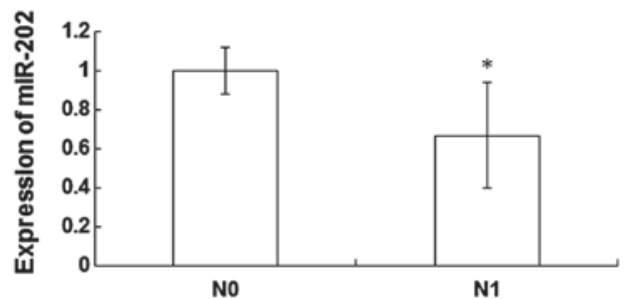

C

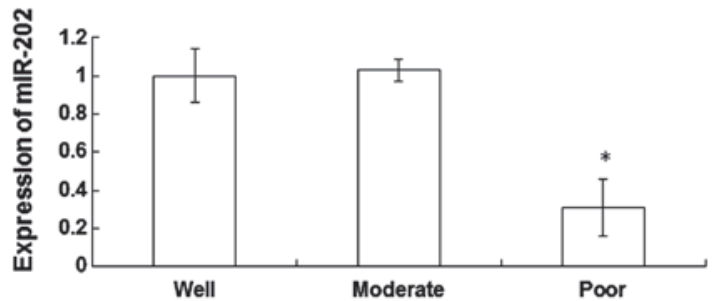

Figure 1. Expression levels of miR-202 in the peripheral blood of patients with esophageal squamous cell carcinoma (ESCC). miR-202 expression levels were detected using reverse transcription-quantitative polymerase chain reaction. Experiments were repeated $>3$ times. Histograms demonstrating (A) the mean normalized expression levels of miR-202 in the peripheral blood of patients with ESCC and healthy individuals, (B) the N0 and N1 lymph node metastasis group and (C) patients with poor, moderate and well differentiated ESCC tissues. Data are expressed as the mean \pm the standard error of the mean. ${ }^{*} \mathrm{P}<0.05$. N0, no evidence of lymph node metastasis; N1, metastasis to lymph node.

layer were subsequently counted using an inverted microscope (magnification, $\mathrm{x} 10$ ).

Statistical analysis. Results are expressed as the mean \pm standard deviation. Statistical analyses were performed using SPSS 11.0 for Windows (SPSS Inc., Chicago, IL, USA). Paired t-test was used to analyze comparisons between the groups and paired data. $\mathrm{P}<0.05$ was considered to indicate a significantly significant difference.

\section{Results}

Expression levels of miR-202 are significantly decreased in the peripheral blood of patients with ESCC. In order to detect the expression levels of miR-202 in the peripheral blood of patients with ESCC and healthy individuals, RT-qPCR was performed. The expression levels of miR-202 were significantly reduced $(0.45 \pm 0.17$ fold; $\mathrm{P}<0.05)$ in the peripheral blood cells of patients with ESCC, as compared with the healthy individuals (Fig. 1A). Furthermore, the expression levels of miR-202 were significantly reduced $(0.67 \pm 0.27$ fold; $\mathrm{P}<0.05)$ in the $\mathrm{N} 1$ lymph node metastasis group, as compared with the N0 lymph node metastasis group (Fig. 1B). Notably, the expression levels of miR-202 were significantly decreased in patients with poorly differentiated ESCC tissues, as compared

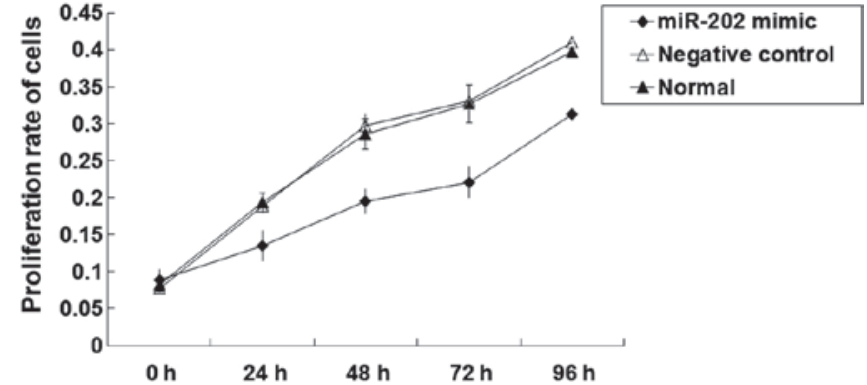

Figure 2. Effect of miR-202 expression on the proliferative ability of EC9706 esophageal squamous cell carcinoma cells, as detected by methylthiazolyl-tetrazolium bromide proliferation assay. Proliferative cells were represented by the optical density of EC9706 cells transfected with miR-202 mimics, negative control and normal EC9706 cells, as determined by a microplate reader at $490 \mathrm{~nm}$. Experiments were repeated $>3$ times.

with patients with well and moderately differentiated ESCC tissues $(\mathrm{P}<0.05)$. Furthermore, miR-202 expression levels were significantly reduced $(0.31 \pm 0.15$ fold; $\mathrm{P}<0.05)$ in patients with poorly differentiated ESCC tissues, as compared with patients with well differentiated ESCC tissues (Fig. 1C). These results indicate that the expression levels of miR-202 are significantly decreased in the peripheral blood of patients with ESCC, which may be associated with the degree of differentiation and lymph node metastasis.

Proliferation of EC9706 cell lines is inhibited by the expression of $m i R-202$. In order to investigate the effects of miR-202 expression levels on cell proliferation, an MTT proliferation assay was performed. As outlined in Fig. 2, the proliferation rates of the negative control and normal EC9706 cells were normal. An 8-fold increase in the number of EC9706 cells was about detected following $96 \mathrm{~h}$ culture $(\mathrm{P}<0.05)$. Following transfection with miR-202 mimics, the proliferation of EC9706 cells gradually increased. As compared with pre-transfection cells, the number of EC9706 cells significantly increased by $<2$-fold at $96 \mathrm{~h}$ following transfection $(\mathrm{P}<0.05)$. These results suggest that the proliferation of EC9706 ESCC cell lines may be inhibited by the expression of miR-202.

Migratory ability of EC9706 ESCC cell lines is inhibited by the expression of $m i R-202$. In order to determine the effects of miR-202 expression levels on the migratory ability of ESCC cells, an in vitro scratch assay was performed. The scratched areas of the negative control and normal EC9706 cells were decreased $24 \mathrm{~h}$ post-scratch, as compared with $0 \mathrm{~h}$ (Fig. 3A and B). However, no significant difference in the scratched area of EC9706 cells transfected with miR-202 mimics was detected following $24 \mathrm{~h}$, as compared with $0 \mathrm{~h}$ (Fig. 3C). These results demonstrated that the migratory ability of EC9706 cells was reduced following transfection with miR-202 mimics, indicating that the migratory ability of ESCC cells may be inhibited by the expression of miR-202.

Invasive ability of EC9706 ESCC cell lines is inhibited by the expression of $m i R-202$. In order to determine the effects of miR-202 expression levels on the invasive ability of EC9706 cells, a Transwell ${ }^{\circledR}$ chamber assay was performed. The number of negative control and normal EC9706 cells 


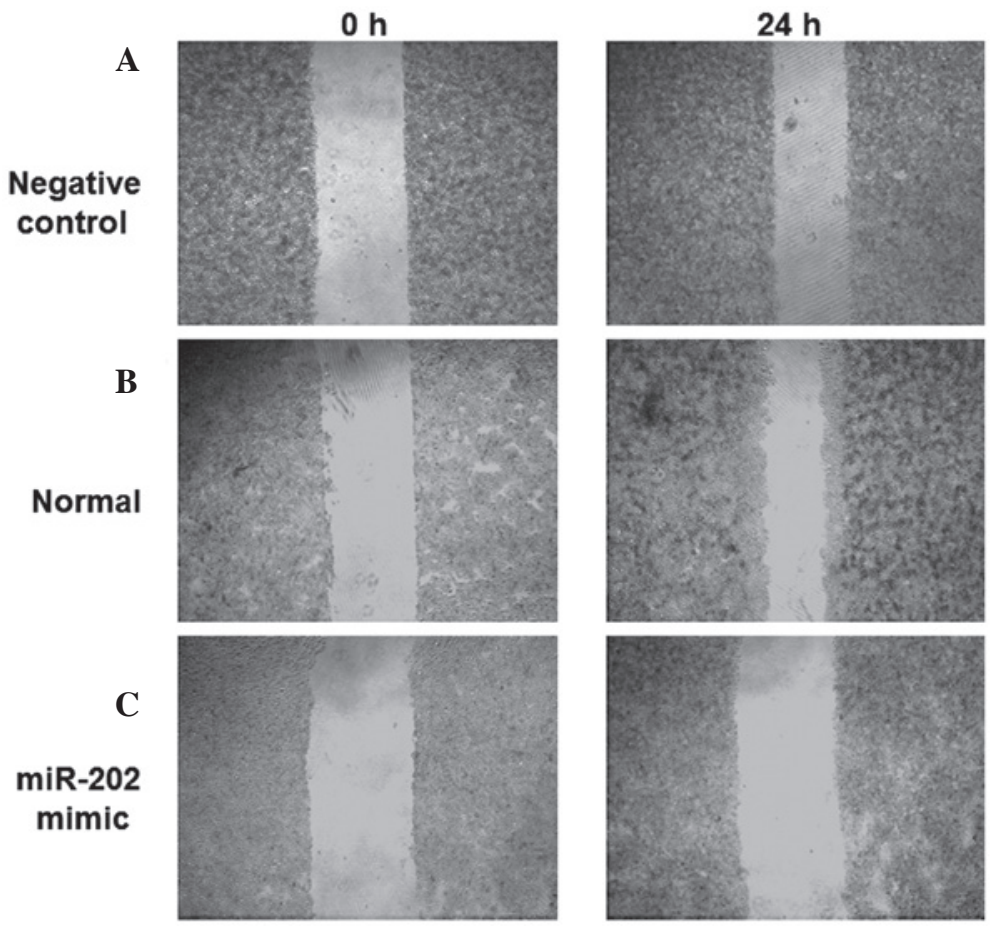

Figure 3. Effect of miR-202 expression on the migratory ability of esophageal squamous cell carcinoma EC9706 cell lines was investigated using an in vitro scratch assay. Cell monolayers were scratched and cultured in RPMI-1640 medium with 10\% fetal bovine serum for $24 \mathrm{~h}$. Scratched areas of (A) negative control, (B) normal EC9706 cells and (C) EC9706 cells transfected with miR-202 mimics were visualized using an inverted microscope at 0 and $24 \mathrm{~h}$ post-scratch (magnification, $\mathrm{x} 10$ ).

A

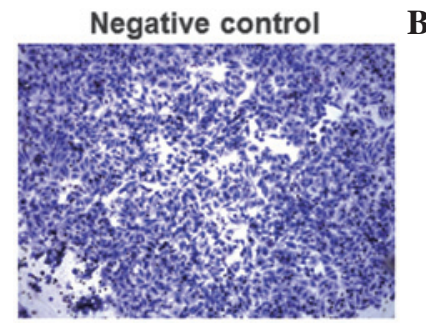

B

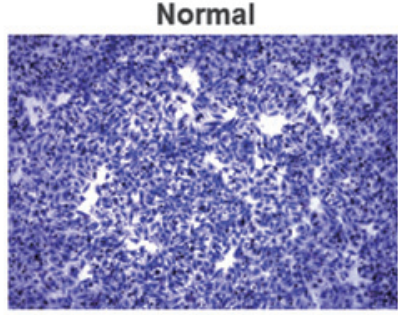

C

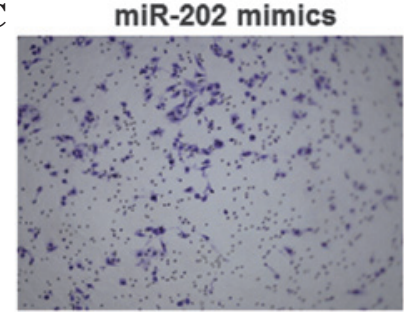

\section{D}

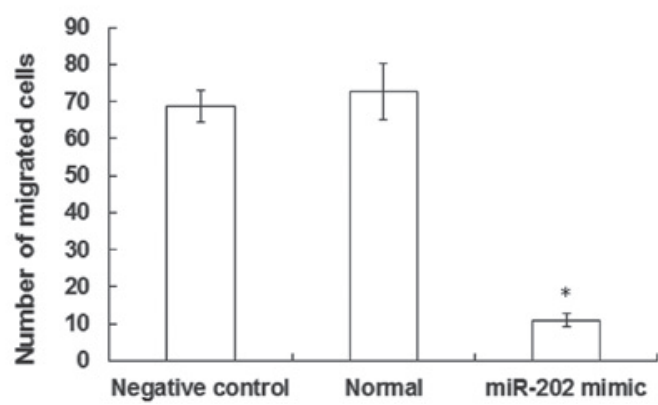

Figure 4. Effect of miR-202 expression on the invasive ability of esophageal squamous cell carcinoma EC 9706 cell lines was investigated using a Transwell ${ }^{\circledR}$ chamber assay. Cells invading through the Matrigel were stained with Giemsa. Random fields of (A) negative control, (B) normal EC9706 cells and (C) EC9706 cells transfected with miR-202 mimics were visualized using an inverted microscope (magnification, x10). (D) Histograms demonstrated the mean normalized number of cells that successfully invaded the Matrigel layer. $\mathrm{P}<0.05$ vs. the negative control.

(Fig. 4A and B) that successfully invaded the Matrigel layer was increased, as when compared with EC9706 cells transfected with miR-202 mimics (Fig. 4C). In particular, the number of cells that invaded the Matrigel layer was 7 fold higher in the negative control group, as compared with the EC9706 cells transfected with miR-202 mimics, a result that was significantly different (Fig. 4D). The results of the Transwell ${ }^{\circledast}$ chamber assay suggest that the invasive ability of ESCC cell lines may be inhibited by the expression of miR-202.

\section{Discussion}

Numerous genes are associated with the development, invasion and metastasis of ESCC. Previous studies have demonstrated that oncogenes, such as p53, c-Myc, matrix metallopepti- 
dase 9 and human sterile alpha motif domain-containing 9, are abnormally expressed in ESCC tissues (22-24). Furthermore, increased expression levels of transforming growth factor- $\beta$ may stimulate the PTEN/PI3K pathway and promote epithelial-mesenchymal transition in the human esophageal epithelium (25). Epithelial-mesenchymal transition is a risk factor for ESCC, as cell junctions are disrupted and the invasive ability of ESCC is enhanced (26).

It has previously been demonstrated that there are evident alterations in the miRNA expression profiles of ESCC tissues and peripheral blood (27). The expression levels of miR-145, miR-143 and miR-21, which were associated with the differentiation, invasion and metastasis of ESCC, have demonstrated clinical significance in the diagnosis and prognosis of patients with ESCC (28). In the present study, RT-qPCR was performed in order to determine the expression levels of miR-202 in the peripheral blood of patients with ESCC. Furthermore, in vitro proliferation, migration and invasion assays were performed to investigate the biological function of miR-202 in the progression of ESCC, which may be useful in the early diagnosis and prognosis of patients with ESCC.

The results of the present study demonstrated that the expression levels of miR-202 were significantly decreased in the peripheral blood of patients with ESCC, which may be associated with the degree of differentiation and lymph node metastasis. Furthermore, the proliferative, migratory and invasive abilities of ESCC cells were inhibited by the expression of miR-202. These results suggested that low miR-202 expression levels in peripheral blood may increase the risk of metastasis in patients with ESCC.

In conclusion, miR-202 expression levels are reduced in the peripheral blood of patients with ESCC. As miR-202 is capable of inhibiting the proliferation, migration and invasion of ESCC cells, assessing miR-202 expression levels may facilitate early diagnosis of ESCC and thus improve the prognosis of patients with ESCC.

\section{Acknowledgements}

The authors thank Professor Lijuan Liu at the Department of Clinical Laboratory (Laiwu People's Hospital, Laiwu, China).

\section{References}

1. Xing SZ and Zhang Y: Efficacy and safety of transdermal fentanyl for the treatment of oral mucositis pain caused by chemoradiotherapy in patients with esophageal squamous cell carcinoma. Support Care Cancer 23: 753-759, 2015.

2. Guo XF, Mao T, Gu ZT, Ji CY, Fang WT and Chen WH: Clinical study on postoperative recurrence in patients with $\mathrm{pN} 0$ esophageal squamous cell carcinoma. J Cardiothorac Surg 9: $150,2014$.

3. Jing W, Zhu H, Guo H, Zhang Y, Shi F, Han A, Li M, Kong L and Yu J: Feasibility of elective nodal irradiation (ENI) and involved field irradiation (IFI) in radiotherapy for the elderly patients (aged $\geq 70$ years) with esophageal squamous cell cancer: A retrospective analysis from a single institute. PLoS One 10: e0143007, 2015.

4. Liu X, Song N, Liu Y, Liu Y, Li J, Ding J and Tong Z: Efficient induction of anti-tumor immune response in esophageal squamous cell carcinoma via dendritic cells expressing MAGE-A3 and CALR antigens. Cell Immunol 295: 77-82, 2015.
5. Song B, Cui H, Li Y, Cheng C, Yang B, Wang F, Kong P, Li H, Zhang L, Jia Z, et al: Mutually exclusive mutations in NOTCH1 and PIK3CA associated with clinical prognosis and chemotherapy responses of esophageal squamous cell carcinoma in China. Oncotarget. doi: 10.18632/oncotarget.6120 [Epub ahead of print], 2015.

6. Arigami T, Okumura H, Matsumoto M, Uchikado Y, Uenosono Y, Kita Y, Owaki T, Mori S, Kurahara H, Kijima Y, et al: Analysis of the fibrinogen and neutrophil-lymphocyte ratio in esophageal squamous cell carcinoma: A promising blood marker of tumor progression and prognosis. Medicine (Baltimore) 94: e1702, 2015.

7. Zhong Y, Xuan P, Han K, Zhang W and Li J: Improved pre-miRNA classification by reducing the effect of class imbalance. Biomed Res Int doi: 10.1155/2015/960108, Epub 2015 Nov 10.

8. Lu Y, Gao W, Zhang C, Wen S, Huangfu H, Kang J and Wang B: Hsa-miR-301a-3p acts as an oncogene in laryngeal squamous cell carcinoma via target regulation of Smad4. J Cancer 6: 1260-1275, 2015.

9. Taccioli C, Garofalo M, Chen H, Jiang Y, Tagliazucchi GM, Di Leva G, Alder H, Fadda P, Middleton J, Smalley KJ, et al: Repression of esophageal neoplasia and inflammatory signaling by anti-miR-31 delivery in vivo. J Natl Cancer Inst 107: pii: djv220, 2015.

10. Meng XR, Lu P, Mei JZ, Liu GJ, and Fan QX: Expression analysis of miRNA and target mRNAs in esophageal cancer. Braz J Med Biol Res 47: 811-817, 2014.

11. Yang M, Liu R, Li X, Liao J, Pu Y, Pan E, Yin L and Wang Y: miRNA-183 suppresses apoptosis and promotes proliferation in esophageal cancer by targeting PDCD4. Mol Cells 37: 873-880, 2014.

12. Okumura T, Shimada Y, Moriyama M, Takei Y, Omura T, Sekine S, Nagata T, Shimizu K and Tsukada K: MicroRNA-203 inhibits the progression of esophageal squamous cell carcinoma with restored epithelial tissue architecture in vivo. Int J Oncol 44: 1923-1932, 2014

13. Yu X, Jiang X, Li H Guo L, Jiang W and Lu SH: miR-203 inhibits the proliferation and self-renewal of esophageal cancer stem-like cells by suppressing stem renewal factor Bmi-1. Stem Cells Dev 23: 576-585, 2014.

14. Sakai NS, Samia-Aly E, Barbera M, and Fitzgerald RC. A review of the current understanding and clinical utility of miRNAs in esophageal cancer. Semin Cancer Biol 23: 512-521, 2013.

15. Gadducci A, Sergiampietri C, Lanfredini N, and Guiggi I: Micro-RNAs and ovarian cancer: The state of art and perspectives of clinical research. Gynecol Endocrinol 30: 266-271, 2014.

16. Rawlings-Goss RA, Campbell MC and Tishkoff SA: Global population-specific variation in miRNA associated with cancer risk and clinical biomarkers. BMC Med Genomics 7: 53, 2014.

17. Sun Z, Zhang T, Hong H, Liu Q and Zhang H: miR-202 suppresses proliferation and induces apoptosis of osteosarcoma cells by downregulating Gli2. Mol Cell Biochem 397: 277-283, 2014.

18. Zhang Y, Zheng D, Xiong Y, Xue C, Chen G, Yan B and Ye Q: miR-202 suppresses cell proliferation in human hepatocellular carcinoma by downregulating LRP6 post-transcriptionally. FEBS Lett 588: 1913-1920, 2014

19. Yu JJ, Shen XJ, Wang XD and Ju SQ: Effect of miR-202 on the growth of multiple myeloma cells via regulating B cell-activating factor and the underlying mechanism. Zhonghua Zhong Liu Za Zhi 35: 886-891, 2013 (In Chinese).

20. Hu N, Clifford RJ, Yang HH, Wang C, Goldstein AM, Ding T, Taylor PR and Lee MP: Genome wide analysis of DNA copy number neutral loss of heterozygosity $(\mathrm{CNNLOH})$ and its relation to gene expression in esophageal squamous cell carcinoma. BMC Genomics 11: 576, 2010.

21. Livak KJ and Schmittgen TD: Analysis of relative gene expression data using real-time quantitative PCR and the 2(-Delta Delta C(T)) Method. Methods 25: 402-408, 2001.

22. Yin X, Zhang R, Feng C, Zhang J, Liu D, Xu K, Wang X, Zhang S, Li Z, Liu X and Ma H: Diallyl disulfide induces $\mathrm{G} 2 / \mathrm{M}$ arrest and promotes apoptosis through the p53/p21 and MEK-ERK pathways in human esophageal squamous cell carcinoma. Oncol Rep 32: 1748-1756, 2014.

23. Zhong C, Fan L, Yao F, Shi J, Fang W and Zhao H: HMGCR is necessary for the tumorigenecity of esophageal squamous cell carcinoma and is regulated by Myc. Tumour Biol 35: 4123-4129, 2014.

24. Tang S, Zheng X, He L, Qiao L, Jing D, Ni Z, Zeng W and Jian M: Significance of SAMD9 expression in esophageal squamous cell carcinoma. Xi Bao Yu Fen Zi Mian Yi Xue Za Zhi 30: 411-413, 2014 (In Chinese). 
25. Zhang HY, Wang ZQ, Li YY, Wang F, Zeng QR, Gao Y, Xuan XY and Li SS: Transforming growth factor- $\beta 1$-induced epithelial-mesenchymal transition in human esophageal squamous cell carcinoma via the PTEN/PI3K signaling pathway. Oncol Rep 32: 2134-2142, 2014.

26. Niwa Y, Yamada S, Koike M, Kanda M, Fujii T, Nakayama G, Sugimoto H, Nomoto S, Fujiwara M and Kodera Y: Epithelial to mesenchymal transition correlates with tumor budding and predicts prognosis in esophageal squamous cell carcinoma. J Surg Oncol 110: 764-769, 2014.
27. Wen J, Luo K, Liu H, Liu S, Lin G, Hu Y, Zhang X, Wang G, Chen Y, Chen Z, et al: MiRNA expression analysis of pretreatment biopsies predicts the pathological response of esophageal squamous cell carcinomas to neoadjuvant chemoradiotherapy. Ann Surg 2015 Oct 1 [Epub ahead of print].

28. Akagi I, Miyashita M, Ishibashi O, Mishima T, Kikuchi K, Makino H, Nomura T, Hagiwara N, Uchida E and Takizaw T: Relationship between altered expression levels of MIR21, MIR143, MIR145, and MIR205 and clinicopathologic features of esophageal squamous cell carcinoma. Dis Esophagus 24: 523-530, 2011. 